\title{
Research Article \\ Effect of (0001) Strain on the Electronic and Magnetic Properties of the Half-Metallic Ferromagnet $\mathrm{Fe}_{2} \mathrm{Si}$
}

\author{
Wang Chen, ${ }^{1}$ Ruijie Li, ${ }^{2}$ and Yanhui Liu ${ }^{1}$ \\ ${ }^{1}$ State Key Laboratory for Seismic Reduction/Control \& Structural Safety (Cultivation), Guangzhou University, \\ Guangzhou 510006, China \\ ${ }^{2}$ School of Materials Science and Engineering, Guizhou Minzu University, Guiyang 550025, China
}

Correspondence should be addressed to Yanhui Liu; liuyanhui2012@163.com

Received 12 June 2017; Accepted 6 September 2017; Published 11 October 2017

Academic Editor: Marco Cannas

Copyright (C) 2017 Wang Chen et al. This is an open access article distributed under the Creative Commons Attribution License, which permits unrestricted use, distribution, and reproduction in any medium, provided the original work is properly cited.

\begin{abstract}
The electronic and magnetic properties of the half-metallic ferromagnet $\mathrm{Fe}_{2} \mathrm{Si}$ under (0001) strain have been evaluated by the firstprinciples density functional theory method. The spin-up band structure shows that bulk $\mathrm{Fe}_{2} \mathrm{Si}$ has metallic character, whereas the spin-down band structure shows that bulk $\mathrm{Fe}_{2} \mathrm{Si}$ is an $\mathrm{S}$-L indirect band gap of $0.518 \mathrm{eV}$ in the vicinity of Fermi surface. Indirectto-direct band gaps and an unstable-to-stable transition are observed in bulk $\mathrm{Fe}_{2} \mathrm{Si}$ as strain is applied. In the range $-11 \%$ to $11 \%$ (excluding zero strain), bulk $\mathrm{Fe}_{2} \mathrm{Si}$ has stable half-metallic ferromagnetism, the spin polarization at the Fermi surface is $100 \%$, and the magnetic moment of the $\mathrm{Fe}_{2} \mathrm{Si}$ unit cell is $4.0 \mu \mathrm{B}$. The density distribution shows that the spin states of bulk $\mathrm{Fe}_{2} \mathrm{Si}$ mainly come from the $\mathrm{Fe}^{1}-3 \mathrm{~d}$ and $\mathrm{Fe}^{3}-3 \mathrm{~d}$ states, indicating that bulk $\mathrm{Fe}_{2} \mathrm{Si}$ has spin-polarized ferromagnetism. The half-metallic ferromagnetism of bulk $\mathrm{Fe}_{2} \mathrm{Si}$ is mainly caused by $\mathrm{d}-\mathrm{d}$ exchange and $\mathrm{p}-\mathrm{d}$ hybridization, which are not sensitive to strain. It is very important to investigate the effect of changes in the lattice constant on the half-metallic ferromagnetic properties of bulk $\mathrm{Fe}_{2} \mathrm{Si}_{\text {. }}$
\end{abstract}

\section{Introduction}

Half-metallic ferromagnets (HMFs) have attracted much attention because of their potential applications in spintronics [1]. Like a normal ferromagnet, HMFs have two different spin channels [2-4]. They have an energy gap in one spin direction at the Fermi level, whereas the other spin is strongly metallic, which results in complete spin polarization of the conduction electrons. Such materials generally have a high Curie temperature and close to $100 \%$ spin polarization $[5,6]$. Therefore, half-metallic ferromagnetic materials will undoubtedly be an ideal semiconductor spin electron injection source. This shows that half-metallic ferromagnetic research is important and has application prospects. In addition, it will promote the rapid development of semiconductor spin electronics.

Bulk $\mathrm{Fe}_{2} \mathrm{Si}$ has not been well investigated. Chen and Tan [7] used X-ray diffraction (XRD) to study the structure of $\mathrm{Fe}_{2} \mathrm{Si}$ thin films. They found that the film thickness is affected by the base material, and the magnetic, electric, and optical characteristics are affected by the film structure. The structure, lattice parameter, phonon spectrum, and reflectance spectrum of hexagonal $\mathrm{Fe}_{2} \mathrm{Si}$ have been investigated by firstprinciples calculations [8]. The results show that $\mathrm{Fe}_{2} \mathrm{Si}$ is a ferromagnetic material and it has a spin-polarized halfmetal-like band structure. However, there have been no studies associating the magnetism and mechanical properties of $\mathrm{Fe}_{2} \mathrm{Si}$. Therefore, in the present work, the properties of bulk $\mathrm{Fe}_{2} \mathrm{Si}$ under (0001) strain were calculated using plane-wave pseudopotential methods based on density functional theory (DFT), and the results were analyzed in detail.

\section{Calculation Method}

There are four $\mathrm{Fe}$ atoms and two $\mathrm{Si}$ atoms in the $\mathrm{Fe}_{2} \mathrm{Si}$ unit cell. The fractional coordinates of the three nonequivalent $\mathrm{Fe}$ atoms are $(0,0,0),(0,0,0.5)$, and $(0.333,0.667,0.78)$. The fractional coordinate of the $\mathrm{Si}$ atoms is $(0.333,0.667,0.28)$. $\mathrm{Fe}_{2} \mathrm{Si}$ belongs to space group $P-3 m 1$ (number 164). The lattice parameters are $a=b=4.052 \AA$ and $c=5.086 \AA$. The crystal plane angles are $\alpha=\beta=90^{\circ}$ and $\gamma=120^{\circ}$. The (0001) strain line changes with the lattice constant $c$ of the hexagonal $\mathrm{Fe}_{2} \mathrm{Si}$ structure with strain ranging from $-12 \%$ to $12 \%$. 
The calculations were performed with the plane-wave pseudopotential method implemented in the Cambridge sequential total energy package [9] to calculate the effect of (0001) strain on the electromagnetic mechanism of half-metallic ferromagnet $\mathrm{Fe}_{2} \mathrm{Si}$. The generalized gradient approximation [10] with the revised approximation of the Perdew-Burke-Ernzerhof scheme was used for the exchange-correlation potential. All of the possible structures were optimized by the Broyden-Fletcher-Goldfarb-Shanno algorithm [11, 12]. Geometry optimization was performed to fully relax the structures until self-consistent field (SCF) convergence per atom. The tolerance in the SCF calculation was $5.0 \times 10^{-6} \mathrm{eV} /$ atom. The ultrasoft pseudopotentials were expanded within a plane-wave basis set with $330 \mathrm{eV}$, and the iteration convergence accuracy was $5.0 \times 10^{-7} \mathrm{eV}$. The energy of bulk $\mathrm{Fe}_{2} \mathrm{Si}$ was calculated based on optimization of the structural system, with the minimum energy structure which was chosen as the stable structure. Sampling of the Brillouin zone (BZ) was performed with an $8 \times 8 \times 6 k$-point mesh according to the Monkhorst-Pack method [13].

\section{Results and Discussion}

\subsection{Electronic Structure}

3.1.1. Band Structure. Figure 1 shows the effect of (0001) strain on the band structure of bulk $\mathrm{Fe}_{2} \mathrm{Si}$ near the Fermi energy. Figure 1(c) shows the band structure without strain. It shows that the band structure of spin-up electrons has metallic character and the band structure of spin-down electrons has semiconductor character. The valence band maximum is $0.164 \mathrm{eV}$ at point $\mathrm{S}$ and the conduction band minimum is $0.682 \mathrm{eV}$ at the $\mathrm{BZ}$ point $\mathrm{L}$. Thus, $\mathrm{Fe}_{2} \mathrm{Si}$ forms a band gap of $0.518 \mathrm{eV}$ in the vicinity of Fermi surface (spin-down). The half-metallic gap [14] is determined by the minimum difference between the lowest energy of the spin conductive bands with respect to the Fermi level and the absolute value of the highest energy of the spin valence bands. Therefore, the half-metallic gap of bulk $\mathrm{Fe}_{2} \mathrm{Si}$ is $0.164 \mathrm{eV}$.

Under (0001) plane strain, the lattice constant strain of bulk $\mathrm{Fe}_{2} \mathrm{Si}$ changes its band structure, which leads to an energy change of the conduction band bottom and the valence band top. The valley near the bottom of the conduction band is divided into two groups of the degenerate valley, forming the conduction band edge and secondary conduction band edge. The energy shift of the valence band changes the light and heavy hole band into two groups of peaks, forming the valence band edge and the secondary valence band edge. The band gap $\left(E_{g}\right)$ of the strain is determined by the heavy cavities and degenerate valleys. Figures 1(a) and 1(b) show the band structure of bulk $\mathrm{Fe}_{2} \mathrm{Si}$ under compressive strain, which changes to a direct band gap of $0.338 \mathrm{eV}$ at point $\mathrm{L}$ (spin-down). The strain reaches $-12 \%$; then bulk $\mathrm{Fe}_{2} \mathrm{Si}$ becomes metallic. Figures $1(\mathrm{~d})-1(\mathrm{f})$ show the band structure under tensile strain, which is the same as the band structure when applying compressive strain. This indicates that, under strain, bulk $\mathrm{Fe}_{2} \mathrm{Si}$ is first a stable half-metallic ferromagnet and then changes to metallic with increasing strain. In conclusion, the band structure of bulk $\mathrm{Fe}_{2} \mathrm{Si}$ can be changed using compressive and tensile strain, verifying that strain is an effective way to control the band structure.

3.1.2. Density of States. Figure 2 shows the total density of states (TDOS) and partial density of states (PDOS) of bulk $\mathrm{Fe}_{2} \mathrm{Si}$ under various strains. The plotted energy range is -6 to $5 \mathrm{eV}$, and lower lying semicore states are omitted for clarity. Only the density of states (DOS) distribution near the Fermi level determines the magnetic properties. Hence, we concentrate on the DOS in the vicinity of the Fermi level, which is set to zero. To investigate the effect of strain on the subelectron structure of the system, the Si-s, Si-p, Fe-s, and Fe-d PDOS were investigated under various strains. The configuration of the extranuclear electrons of $\mathrm{Si}$ is $3 \mathrm{~s}^{2} 3 \mathrm{p}^{2}$, and the configuration of the extranuclear electrons of $\mathrm{Fe}$ is $3 d^{6} 4 s^{2}$. Figure 2 shows that the DOS near the Fermi level mainly comes from $\mathrm{Fe}-3 \mathrm{~d}$ spin states, and the contributions of $\mathrm{Si}-3 \mathrm{p}$ spin states are small. There is large exchange splitting between the spin-up and spin-down bands of the Fe-3d states, which leads to a large localized spin magnetic moment at the $\mathrm{Fe}$ atoms and results in polarization of the Fe-3d bands away from the Fermi level. Hybridization of $3 p$ states provided by $\mathrm{Si}$ atoms with $3 \mathrm{~d}$ electrons determines the degree of occupation of the $\mathrm{p}-\mathrm{d}$ orbitals. Therefore, the half-metallic ferromagnetism of bulk $\mathrm{Fe}_{2} \mathrm{Si}$ under various strains is mainly caused by $\mathrm{d}-\mathrm{d}$ exchange and $\mathrm{p}-\mathrm{d}$ hybridization.

The spin-up DOS is mainly distributed below the Fermi level. The spin-down DOS has two main peaks, which are located on both sides of the Fermi level. The spin-up and spin-down TDOS distributions near the Fermi surface are asymmetric; that is, the number of electrons in the spinup and spin-down is quite different, which is the main contribution to the magnetic properties. Figures 2(b), 2(d), and 2(e) show that the Fermi level is completely located in the band gap of the spin-down energy band and the spin polarization of bulk $\mathrm{Fe}_{2} \mathrm{Si}$ at the Fermi level is $100 \%$, so the spin polarization of bulk $\mathrm{Fe}_{2} \mathrm{Si}$ can be improved by strain.

\subsection{Magnetic Properties}

3.2.1. Magnetic Mechanism Analysis. The magnetic moments of all of the atoms in the $\mathrm{Fe}_{2} \mathrm{Si}$ unit cell under various strains are given in Table 1 . The total magnetic moment of the $\mathrm{Fe}_{2} \mathrm{Si}$ unit cell under strains of $-12 \%,-7 \%, 0 \%, 5 \%$, $9 \%$, and $12 \%$ are $9.29 \mu \mathrm{B}, 4.01 \mu \mathrm{B}, 3.54 \mu \mathrm{B}, 4.00 \mu \mathrm{B}, 4.00 \mu \mathrm{B}$, and $4.05 \mu \mathrm{B}$, respectively. This shows that bulk $\mathrm{Fe}_{2} \mathrm{Si}$ has stable half-metallic ferromagnetism under strain of $-11 \%$ to $11 \%$ (excluding zero strain), which agrees well with Figures 1 and 2. If half-metals are in the same applied magnetic field, larger magnetic moments possibly result in stronger spin-correlation scattering of conductive electrons, and the variation of the resistance is then larger. Therefore, bulk $\mathrm{Fe}_{2} \mathrm{Si}$ under strain of $-12 \%$ and $12 \%$ will have more magnetoresistance than under no strain. Table 1 shows that the magnetic moment of bulk $\mathrm{Fe}_{2}$ Si mainly comes from the $\mathrm{Fe}^{1}-3 \mathrm{~d}$ and $\mathrm{Fe}^{3}-$ $3 \mathrm{~d}$ states. 


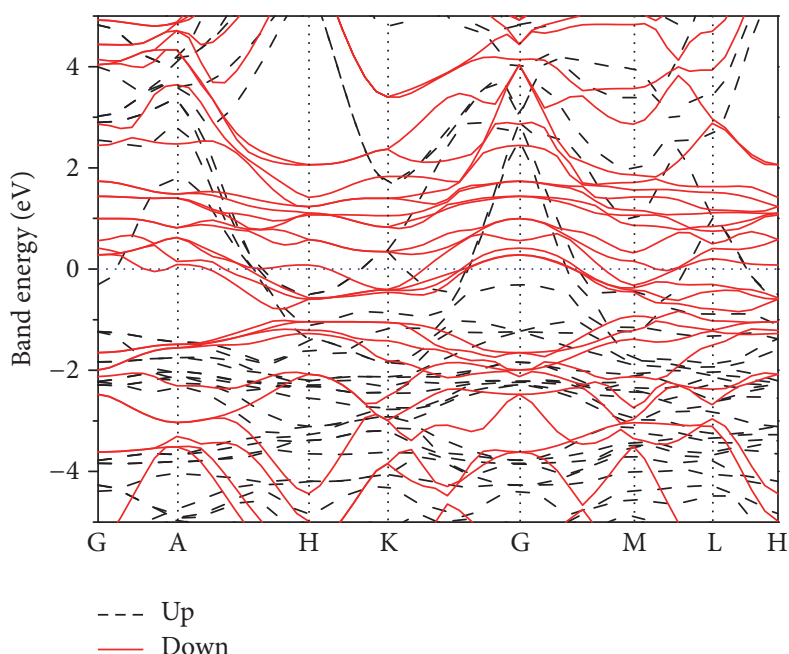

(a)

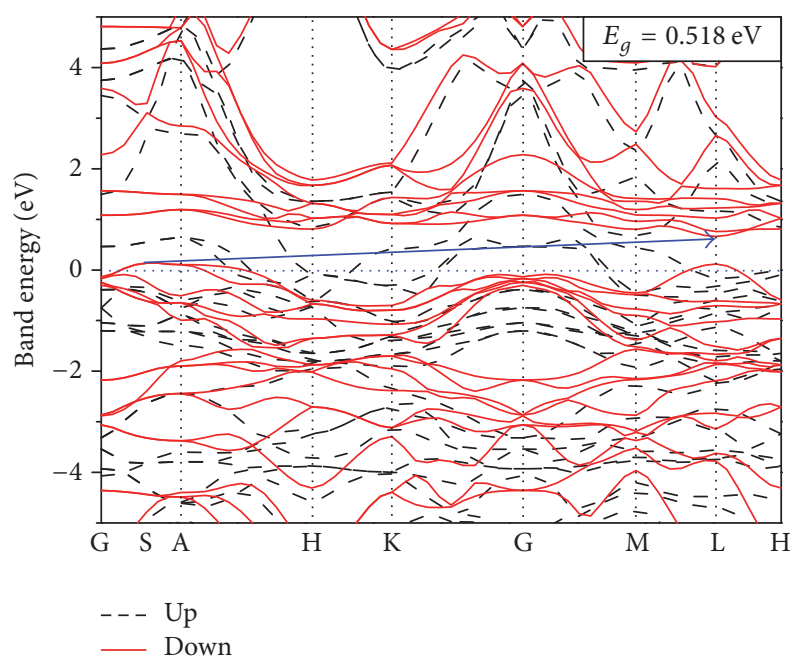

(c)

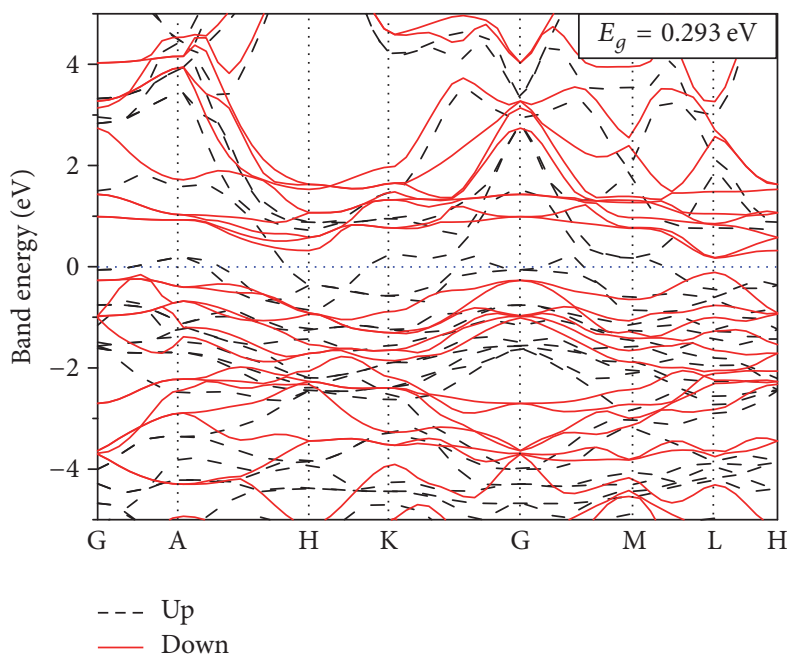

(e)

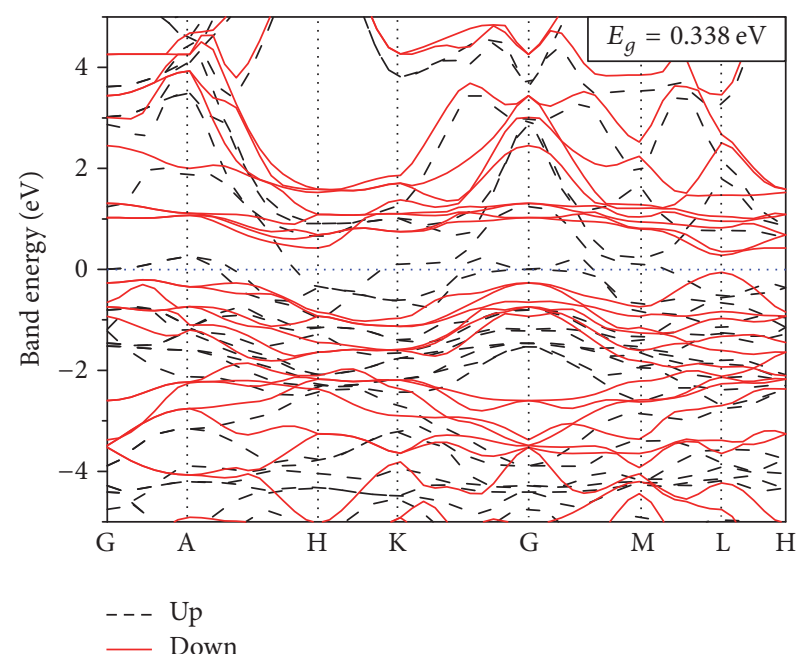

(b)

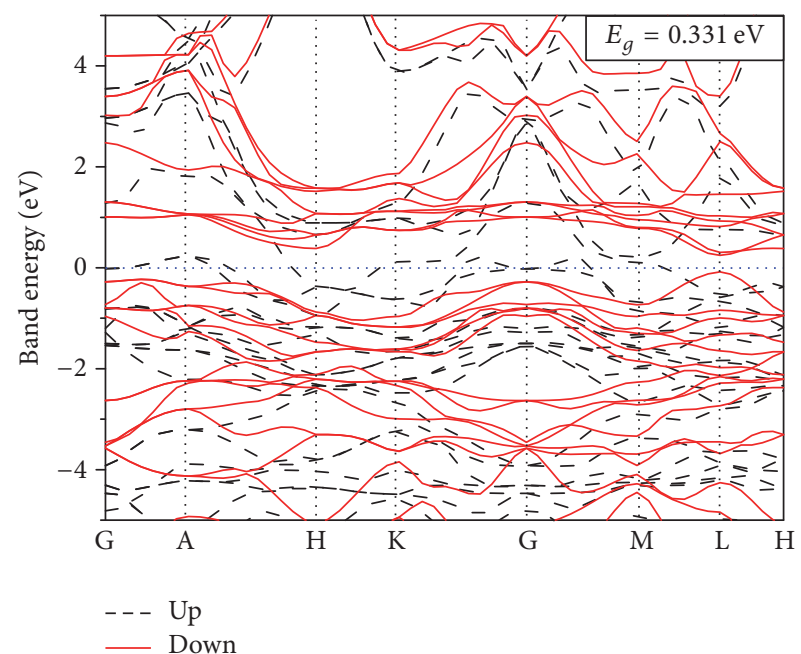

(d)

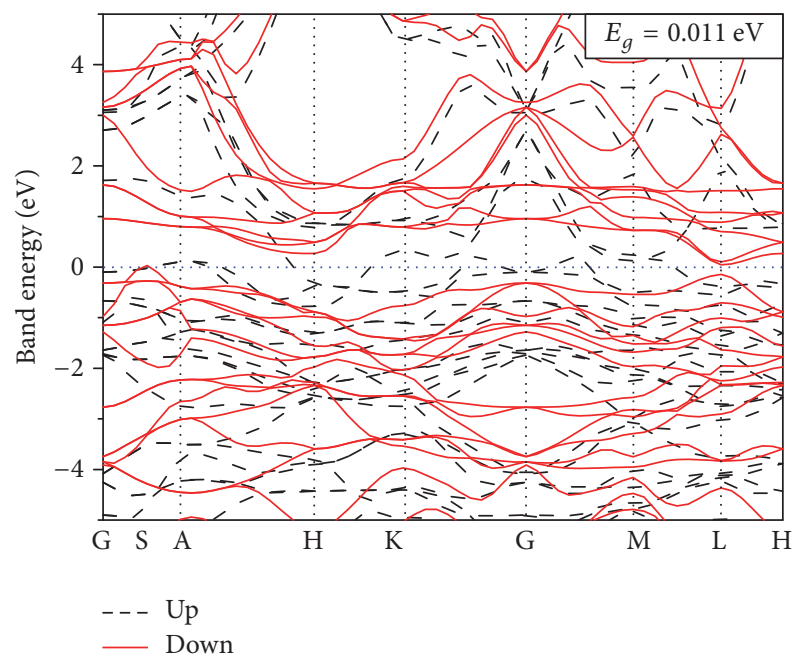

(f)

Figure 1: Band structures of $\mathrm{Fe}_{2} \mathrm{Si}$ under various strains: (a) $-12 \%$, (b) $-7 \%$, (c) $0 \%$, (d) $5 \%$, (e) $9 \%$, and (f) $12 \%$. The band structure of spindown electrons has indirect bandgap semiconductor character. The valence band maximum is $0.164 \mathrm{eV}$ at point $\mathrm{S}$ and the conduction band minimum is $0.682 \mathrm{eV}$ at the $\mathrm{BZ}$ point $\mathrm{L}$. The blue arrow is from the top of the valence band point to the bottom of the conduction band. 


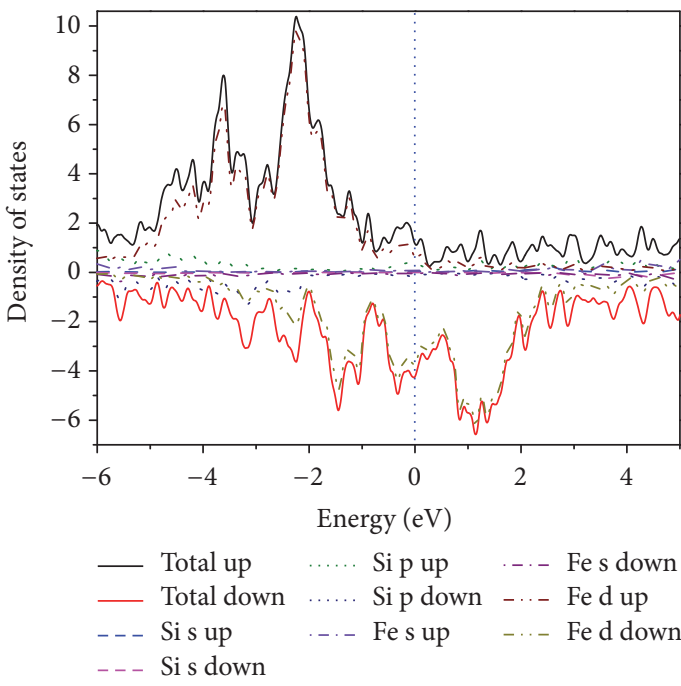

(a)

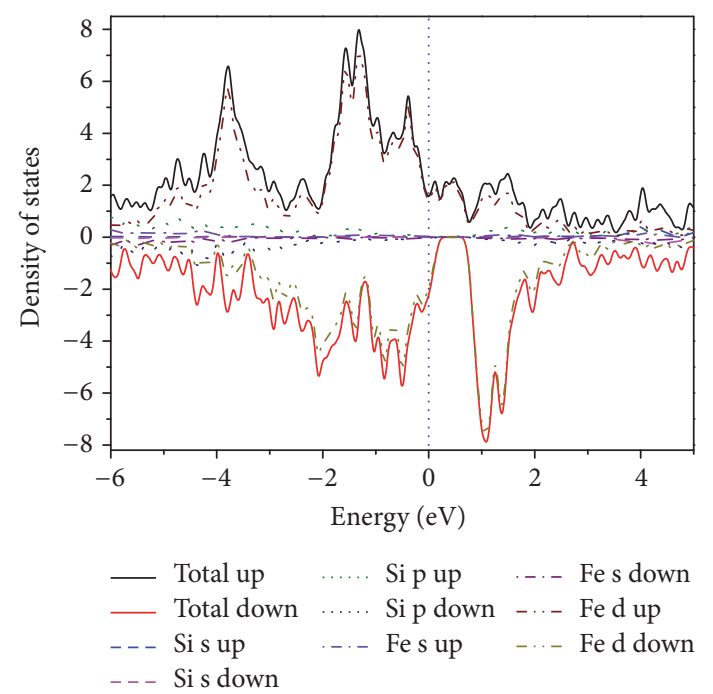

(c)

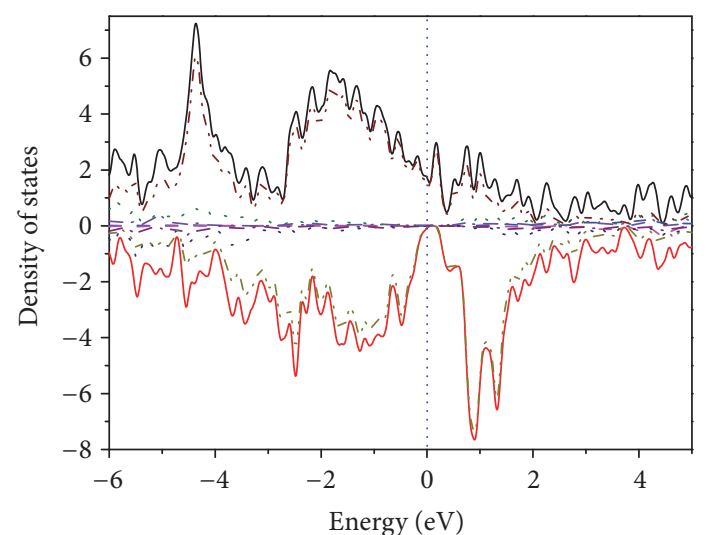

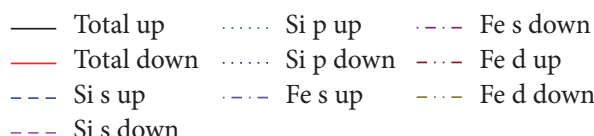

(e)

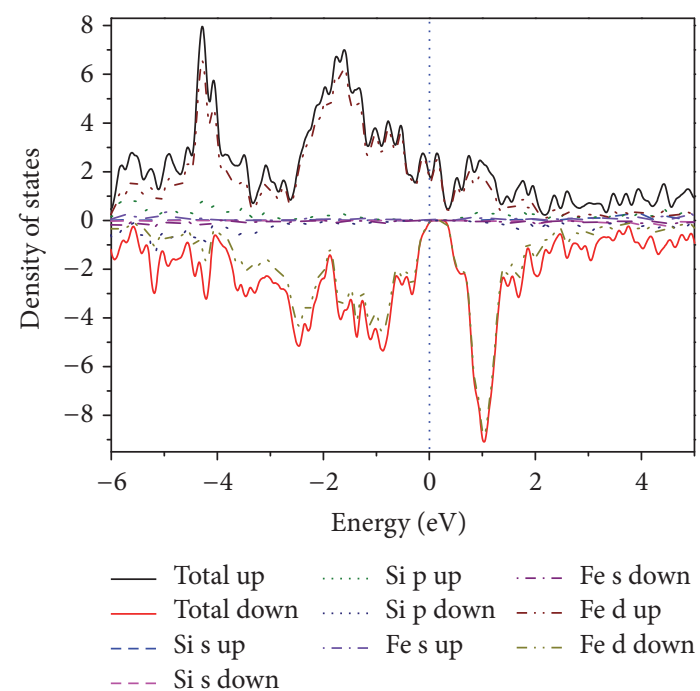

(b)

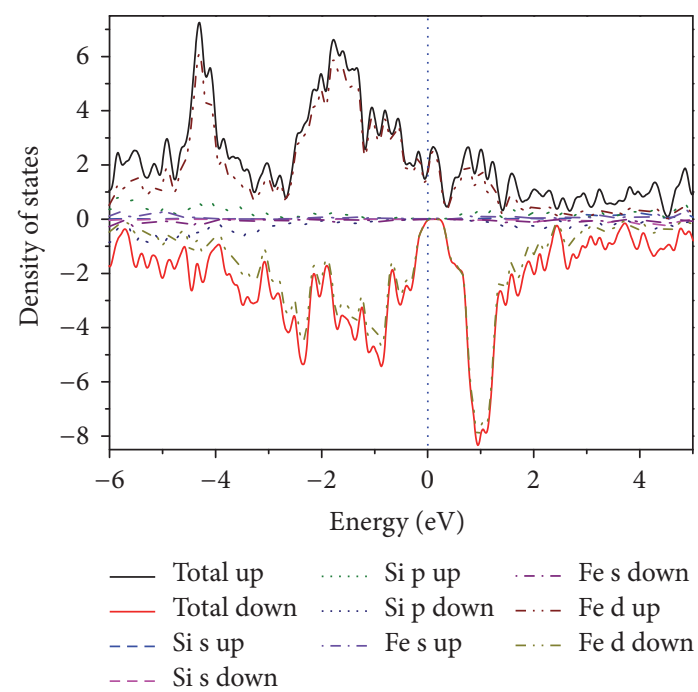

(d)

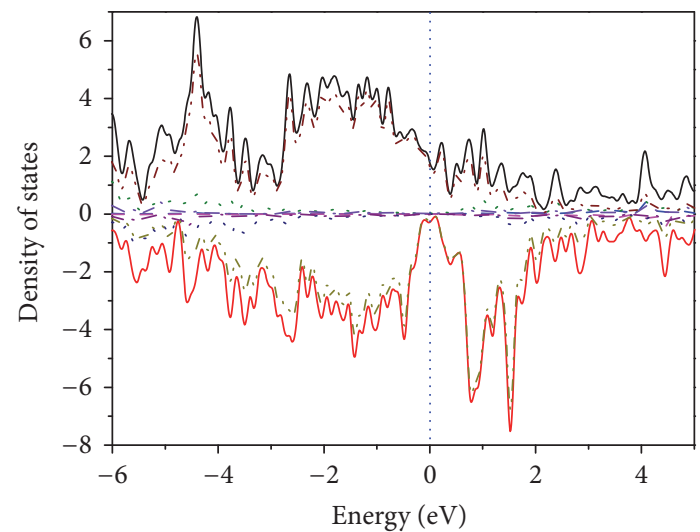

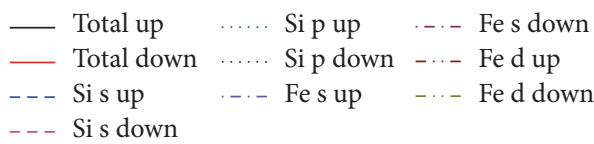

(f)

Figure 2: DOS of $\mathrm{Fe}_{2} \mathrm{Si}$ under various strains: (a) $-12 \%$, (b) $-7 \%$, (c) $0 \%$, (d) $5 \%$, (e) $9 \%$, and (f) $12 \%$. 


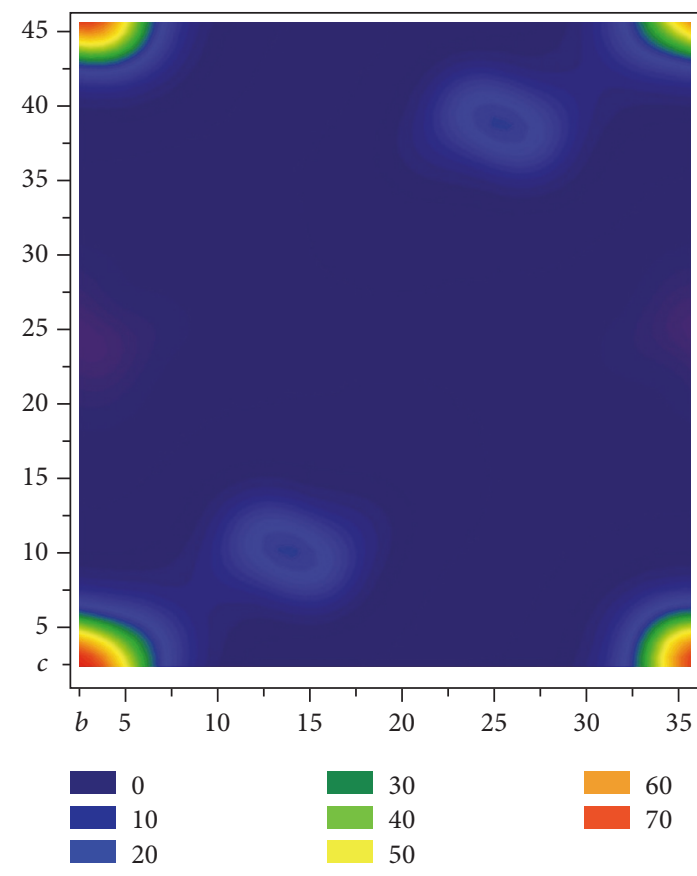

(a)

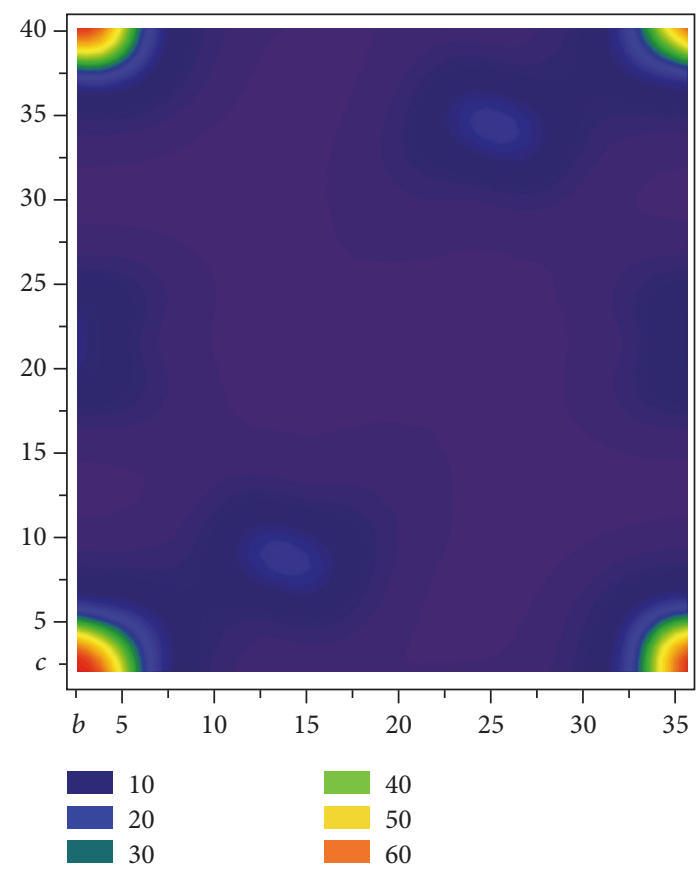

(c)

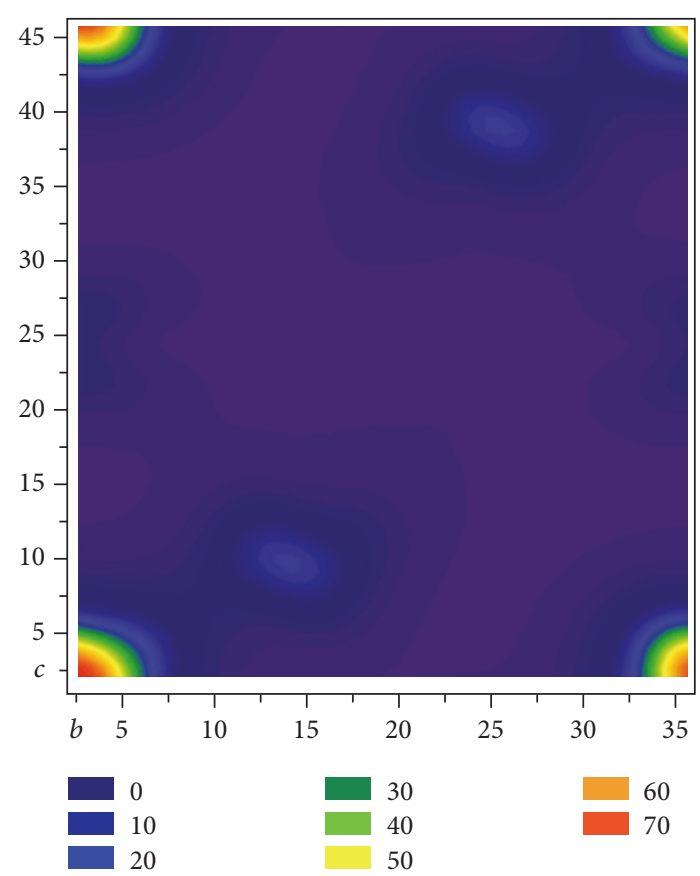

(b)

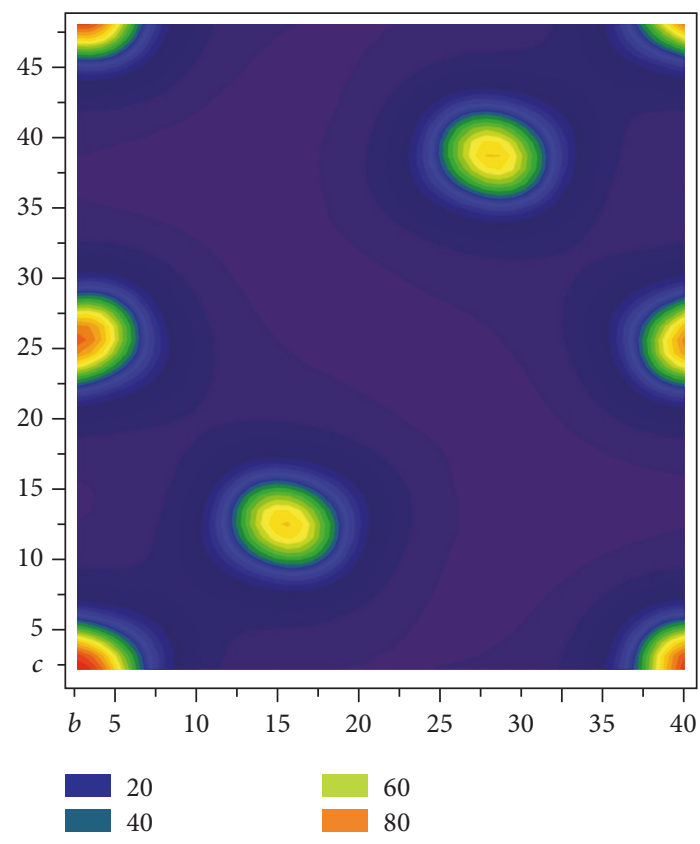

(d)

Figure 3: Spin density distributions of bulk $\mathrm{Fe}_{2} \mathrm{Si}$ under various strains: (a) $0 \%$, (b) $-11 \%$ to $11 \%$ (excluding zero strain), (c) $12 \%$, and (d) $-12 \%$.

In the range $-11 \%$ to $11 \%$ (excluding zero strain), the total magnetic moment of bulk $\mathrm{Fe}_{2} \mathrm{Si}$ is $4.0 \mu \mathrm{B}$ and the spin polarization is $100 \%$ from the DOS map, indicating that the half-metallic ferromagnetic properties of bulk $\mathrm{Fe}_{2} \mathrm{Si}$ are not sensitive to strain. In practical application in an optional electronic device, a small change in the lattice constant may have a significant effect on the electron transport properties at the Fermi level [15]. Therefore, it is very important to investigate the effect of changes in the lattice constant on the half-metallic ferromagnetic properties of bulk $\mathrm{Fe}_{2} \mathrm{Si}$.

3.2.2. Distribution of the Magnetic and Electric Charge. Figure 3 shows the spin density distributions of bulk $\mathrm{Fe}_{2} \mathrm{Si}$ under various strains. The results show that the Fe atoms are surrounded by a high potential, whereas the Si atoms are surrounded by a low potential. The charge of $\mathrm{Fe}_{2} \mathrm{Si}$ under 
TABLE 1: Magnetic moments of all of the atoms in the $\mathrm{Fe}_{2} \mathrm{Si}$ unit cell under various strains.

\begin{tabular}{|c|c|c|c|c|c|c|c|}
\hline Species & Ion & $\begin{array}{c}-12 \% \\
\text { Spin }(\mu \mathrm{B})\end{array}$ & $\begin{array}{c}-7 \% \\
\text { Spin }(\mu \mathrm{B}) \\
\end{array}$ & $\begin{array}{c}0 \% \\
\text { Spin }(\mu \mathrm{B}) \\
\end{array}$ & $\begin{array}{c}5 \% \\
\text { Spin }(\mu \mathrm{B}) \\
\end{array}$ & $\begin{array}{c}9 \% \\
\text { Spin }(\mu \mathrm{B}) \\
\end{array}$ & $\begin{array}{c}12 \% \\
\text { Spin }(\mu \mathrm{B})\end{array}$ \\
\hline $\mathrm{Si}$ & 1 & -0.34 & -0.09 & -0.06 & -0.09 & -0.09 & -0.09 \\
\hline $\mathrm{Si}$ & 2 & -0.34 & -0.09 & -0.06 & -0.09 & -0.09 & -0.09 \\
\hline $\mathrm{Fe}$ & 1 & 2.81 & 2.83 & 2.80 & 2.81 & 2.75 & 2.69 \\
\hline $\mathrm{Fe}$ & 2 & 2.58 & 0.06 & -0.62 & 0.07 & 0.23 & 0.40 \\
\hline $\mathrm{Fe}$ & 3 & 2.29 & 0.65 & 0.74 & 0.65 & 0.60 & 2.57 \\
\hline $\mathrm{Fe}$ & 4 & 2.29 & 0.65 & 0.74 & 0.65 & 0.60 & 2.57 \\
\hline
\end{tabular}

various strains is mainly localized on the Fe atoms and there is little charge on the $\mathrm{Si}$ atoms, indicating that bulk $\mathrm{Fe}_{2} \mathrm{Si}$ has spin polarization ferromagnetism, which is consistent with the results in Table 1 . The charge cloud distribution of the $\mathrm{Si}$ atom is affected by the Fe atom, making the distribution along the (11 $\overline{2} 0)$ crystal face. Charge transfer of the atoms in the system under stress is because of Fe $\mathrm{d}-\mathrm{d}$ exchange and $\mathrm{Fe}-\mathrm{d}-\mathrm{Si}-\mathrm{p}$ hybridization. In the range $-11 \%$ to $11 \%$ (excluding zero strain), the charge density between atoms is relatively low. Figure 3 shows that the spin states of bulk $\mathrm{Fe}_{2} \mathrm{Si}$ mainly come from the $\mathrm{Fe}^{1}-3 \mathrm{~d}$ and $\mathrm{Fe}^{3}-3 \mathrm{~d}$ states. Bulk $\mathrm{Fe}_{2}$ Si maintains its spin polarization ferromagnetism in the strain range.

\section{Conclusion}

The effect of (0001) strain on the electronic structure and magnetic properties of bulk $\mathrm{Fe}_{2} \mathrm{Si}$ has been investigated by the first-principle pseudopotential method based on DFT. The spin-up band structure shows that bulk $\mathrm{Fe}_{2} \mathrm{Si}$ has metallic character, whereas the spin-down band structure shows that bulk $\mathrm{Fe}_{2} \mathrm{Si}$ is an $\mathrm{S}$-L indirect band gap in the vicinity of Fermi surface. The half-metallic gap of bulk $\mathrm{Fe}_{2} \mathrm{Si}$ is $0.164 \mathrm{eV}$. Applying strain, bulk $\mathrm{Fe}_{2} \mathrm{Si}$ is first a stable half-metallic ferromagnet, and it then becomes metallic with further increasing strain. The DOS near the Fermi level mainly comes from Fe-3d spin states, and the DOS of Fe mainly comes from the $\mathrm{Fe}^{1}-3 \mathrm{~d}$ and $\mathrm{Fe}^{3}-3 \mathrm{~d}$ states, which are the source of the ferromagnetic properties of bulk $\mathrm{Fe}_{2} \mathrm{Si}$. The magnetic moment and charge transfer of bulk $\mathrm{Fe}_{2} \mathrm{Si}$ are mainly caused by $\mathrm{Fe} \mathrm{d}-\mathrm{d}$ exchange and $\mathrm{Fe}-\mathrm{d}-\mathrm{Si}-\mathrm{p}$ hybridization. In the strain range $-11 \%$ to $11 \%$ (excluding zero strain), bulk $\mathrm{Fe}_{2} \mathrm{Si}$ has stable half-metallic ferromagnetism and the spin polarization at the Fermi level is $100 \%$, indicating that the spin polarization and half-metallic ferromagnetism of bulk $\mathrm{Fe}_{2} \mathrm{Si}$ can be improved by strain.

\section{Conflicts of Interest}

The authors declare that they have no conflicts of interest.

\section{Acknowledgments}

This work was mainly supported by the Cultivated Project of Outstanding Young Teachers of Guangdong Province (no. YQ2015124). This support is greatly appreciated.

\section{References}

[1] H. Ohno, "Making nonmagnetic semiconductors ferromagnetic," Science, vol. 281, no. 5379, pp. 951-956, 1998.

[2] W.-H. Xie and B.-G. Liu, "Half-metallic ferromagnetlsm in ternary transltion-metal compounds based on ZnTe and CdTe semiconductors," Journal of Applied Physics, vol. 96, no. 6, pp. 3559-3561, 2004.

[3] R. Y. Oeiras, F. M. Araújo-Moreira, and E. Z. Da Silva, "Defectmediated half-metal behavior in zigzag graphene nanoribbons," Physical Review B - Condensed Matter and Materials Physics, vol. 80, no. 7, Article ID 073405, 2009.

[4] K. Hasegawa, M. Isobe, T. Yamauchi et al., "Discovery of ferromagnetic-half-metal-to-insulator transition in K2Cr8O16," Physical Review Letters, vol. 103, no. 14, Article ID 146403, 2009.

[5] R. A. De Groot, F. M. Mueller, P. G. V. Engen, and K. H. J. Buschow, "New class of materials: Half-metallic ferromagnets," Physical Review Letters, vol. 50, no. 25, pp. 2024-2027, 1983.

[6] S. M. Watts, S. Wirth, S. Von Molnár, A. Barry, and J. M. D. Coey, "Evidence for two-band magnetotransport in halfmetallic chromium dioxide," Physical Review B - Condensed Matter and Materials Physics, vol. 61, no. 14, pp. 9621-9628, 2000.

[7] Y. T. Chen and Y. C. Tan, "The optical, magnetic, and electrical characteristics of $\mathrm{Fe}_{2} \mathrm{Si}$ thin films," Journal of Alloys and Compounds, vol. 615, pp. 946-949, 2014.

[8] C. P. Tang, K. V. Tam, S. J. Xiong, J. Cao, and X. Zhang, "The structure and electronic properties of hexagonal Fe2Si," AIP Advances, vol. 6, no. 6, Article ID 065317, 2016.

[9] M. D. Segall, P. J. D. Lindan, M. J. Probert et al., "First-principles simulation: ideas, illustrations and the CASTEP code," Journal of Physics Condensed Matter, vol. 14, no. 11, pp. 2717-2744, 2002.

[10] J. P. Perdew, K. Burke, and M. Ernzerhof, "Generalized gradient approximation made simple," Physical Review Letters, vol. 77, no. 18, pp. 3865-3868, 1996.

[11] C. G. Broyden, "The convergence of a class of double-rank minimization algorithms. II. The new algorithm," Journal of the Institute of Mathematics and Its Applications, vol. 6, pp. 222-231, 1970.

[12] D. Goldfarb, "A family of variable-metric methods derived by variational means," Mathematics of Computation, vol. 24, pp. 23-26, 1970.

[13] H. J. Monkhorst and J. D. Pack, "Special points for Brillouinzone integrations," Physical Review. B. Solid State, vol. 13, no. 12, pp. 5188-5192, 1976.

[14] W. Xie, Y. Xu, B. Liu, and D. G. Pettifor, "Erratum: Half-metallic ferromagnetism and structural stability of zincblende phases 
of the transition-metal chalcogenides," Physical Review Letters, vol. 91, no. 21, 2003.

[15] T. Block, M. J. Carey, B. A. Gurney, and O. Jepsen, "Bandstructure calculations of the half-metallic ferromagnetism and structural stability of full- and half-Heusler phases," Physical Review B - Condensed Matter and Materials Physics, vol. 70, no. 20, Article ID 205114, pp. 1-205114, 2004. 

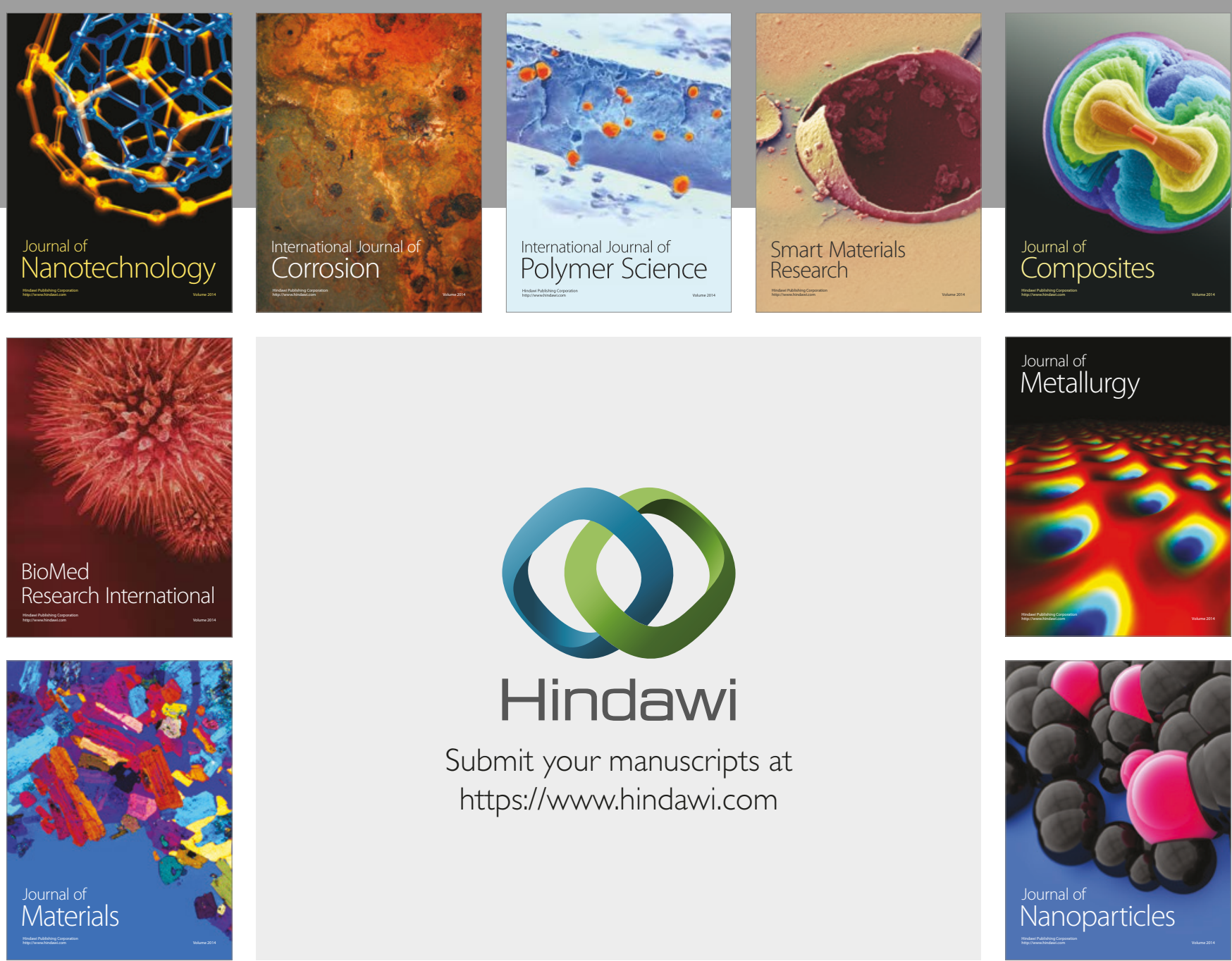

\section{Hindawi}

Submit your manuscripts at

https://www.hindawi.com
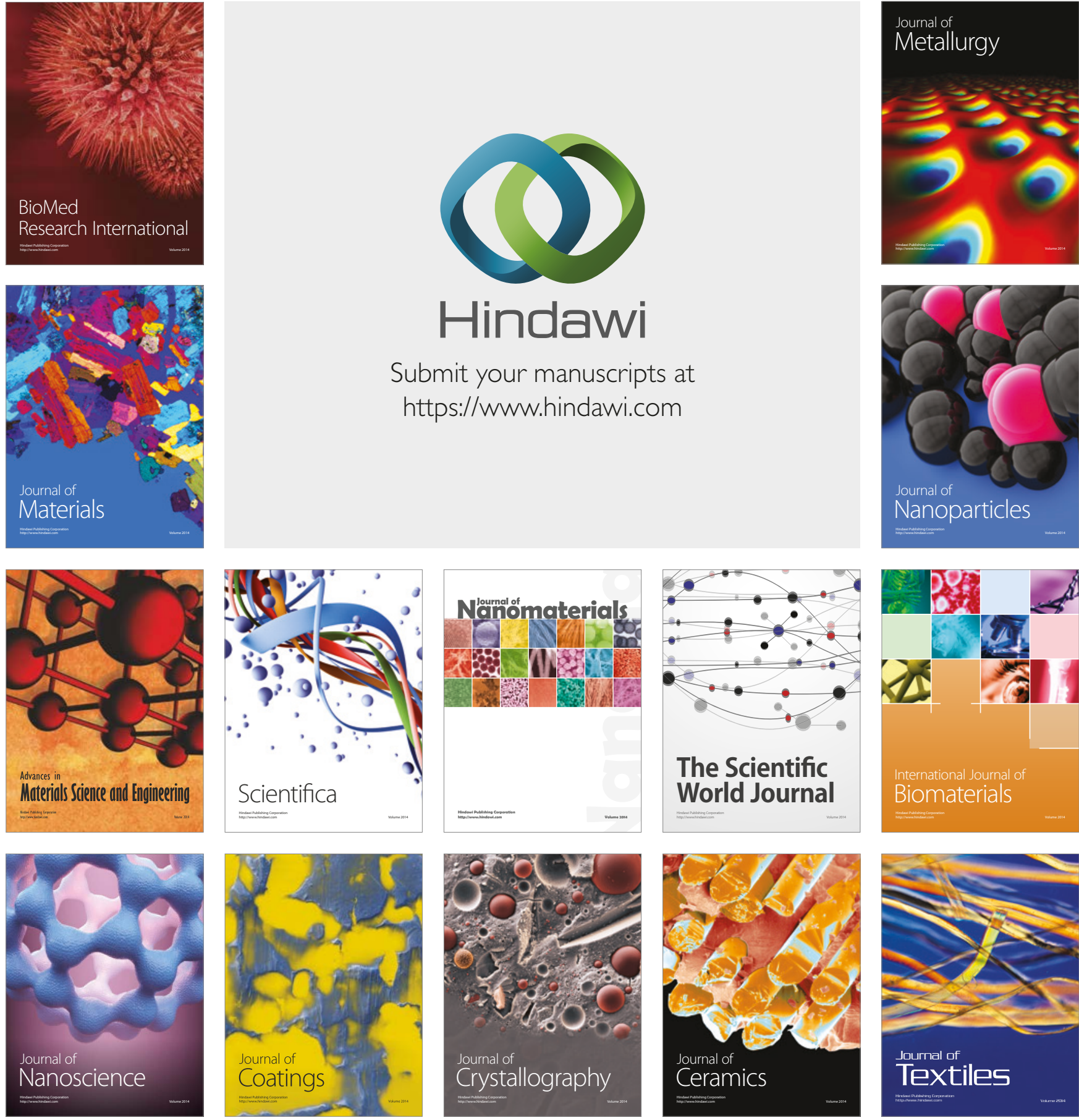

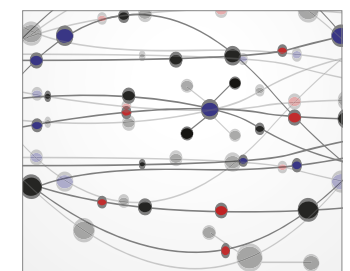

The Scientific World Journal
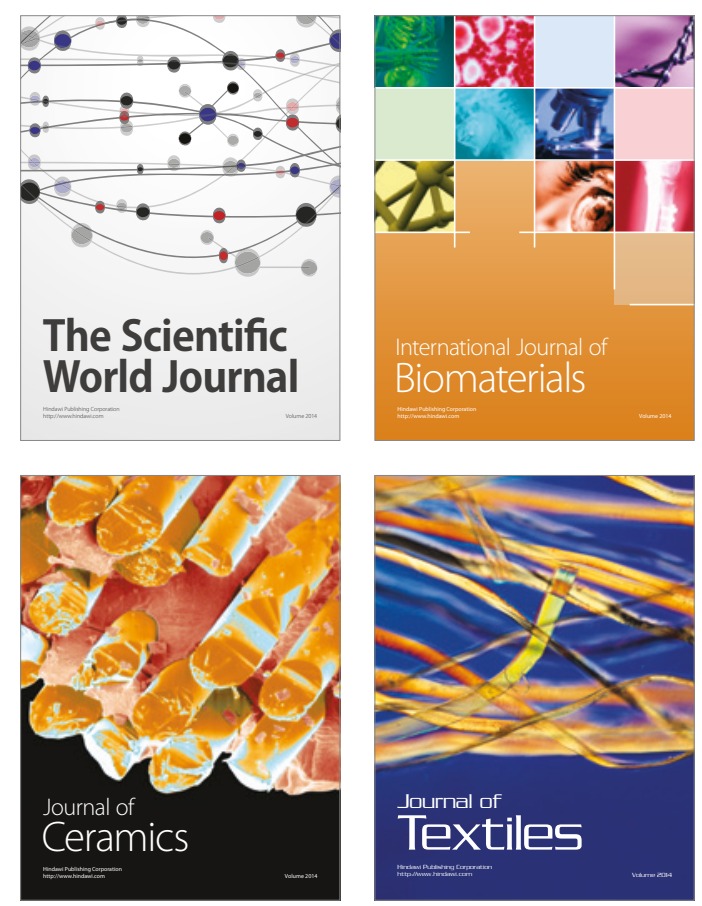